\title{
Evaluation of Annexin V and Calcein-AM as Markers of Mononuclear Cell Apoptosis During Human Immunodeficiency Virus Infection
}

Priscila F.R. Palma ${ }^{1}$, Giovana L. Baggio ${ }^{2}$, Celso Spada ${ }^{1}$, Renata da Silva ${ }^{3}$, Silvia Inês A.C.P. Ferreira ${ }^{3}$ and Aricio Treitinger ${ }^{1}$

${ }^{1}$ Department of Clinical Analysis, Federal University of Santa Catarina (UFSC); Florianópolis, SC; ${ }^{2}$ Division of Infectious Diseases, Federal University of São Paulo (UNIFESP/EPM); São Paulo, SP; ${ }^{3}$ Santa Luzia Medical Laboratory; Florianópolis, SC, Brazil

\begin{abstract}
Evaluation of apoptosis by flow cytometry is generally accomplished by methods that use annexin V-FITC as vital dye, which access phosphatidylserine exposed on the external membrane at the beginning of this process. In addition, the concomitant use of propidium iodide makes possible to verify the characteristic nuclear alterations in the late stages of apoptosis, as a consequence of the increase in membrane permeability. On the other hand, the use of calcein-AM in association with ethidium homodimer (EthD-1) allows the evaluation of cell apoptosis through detection of esterase activity and cellular membrane physical and chemical alterations. The aim of this study was to compare the sensibility of calcein-AM and EthD-1 with annexin V-FITC and propidium iodide for early apoptosis evaluation in peripheral blood mononuclear cell culture, obtained from HIV-infected patients. Apoptosis and cellular viability were detected and quantified by flow cytometry after 24 and 48 hours incubation times. Our results showed that calcein-AM/EthD-1 was more sensitive for apoptotic cell quantification in both incubation times than annexin V-FITC/propidium iodide (mean of $46.95 \% \pm 3.56$, $p<0.0001$, for 24 hours and mean of $37.67 \% \pm 2.47, p<0.0014$ for 48 hours), besides allowing to clearly define viable, apoptotic and dead cell populations.
\end{abstract}

Key-Words: Apoptosis, flow cytometry, annexin V, propidium iodide, calcein-AM, ethidium homodimer.

Abbreviations: Calcein-AM, calcein acetoxymethyl ester; EthD-1, ethidium homodimer; FITC, fluorescein isothiocyanate.

Programmed cell death or apoptosis is histologically characterized by chromatin condensation, internucleosomal cleavage of genomic DNA, plasma membrane blebbing, and fragmentation into apoptotic bodies [1,2].

Apoptosis plays a complementary but opposite role in cell division as a homeostatic mechanism in the morphogenesis and normal tissue reconstruction [3,4]. Equally, this process is important for removing damaged, infected or potentially neoplasic cells [5]. However, both too little and too much apoptotic cell death can lead to adverse biological consequences [6,7]. Rheumatoid arthritis and different cancer types are the best examples for too little apoptosis. Ischemic heart disease, AIDS and neurodegenerative diseases such as Alzheimer's and Parkinson's are the best examples for too much apoptosis $[8,9]$.

Precise analysis and reliable quantification of apoptotic cells are possible through cytofluorimetric approaches, taking advantages of the multiple cellular alterations occurring during apoptosis [10,11]. For example, the reduction in cell volume and increase in cell granularity of apoptotic cells modify their light scattering properties, i.e. the forward scatter (FSC) and side scatter (SSC) parameters respectively [12].

Annexin $\mathrm{V}$ was shown to be useful for detection of apoptotic cells because of its binding affinity for negatively

Received on 5 December 2007; revised 4 April 2008.

Address for correspondence: Dr. Arício Treitinger. Universidade Federal de Santa Catarina, Centro de Ciências da Saúde, Departamento de Análises Clínicas, Campus Universitário - Trindade. Cx. P. 476. Zip code: 88.010-970 - Florianópolis - Santa Catarina - Brazil. Phone: +55 48 3721-9712 Fax: +55 48 3721-9542. E-mail: aricio@ccs.ufsc.br.

The Brazilian Journal of Infectious Diseases Publishing. All rights reserved. charged phospholipids like phosphatidylserine, exposed at the beginning of apoptotic process [13-15]. The conjugation between fluorescein isothiocyanate (FITC) and annexin V allows identifying and quantifying the apoptotic cells by flow cytometry $[13,16]$. Therefore, the concomitant use of the nuclear fluorescent dye propidium iodide (PI) makes possible to verify the nuclear alterations, characteristic of the late stages of the apoptosis [17]. Such property is due to the fact that high molecular weight DNA binding dyes, such as propidium iodide, can not enter intact cell because of their large size and can not either stain apoptotic cells without permeability alterations in plasma membrane, which happen in the final stages of apoptosis. Thus, using flow cytometry, annexin V-FITC allows detecting the initial apoptosis stages, while the propidium iodide allows evaluating the final moments of this cellular death process [16].

The acetoxymethyl ester of calcein is a lipid-soluble fluorogenic diester that passively crosses the cell membrane in an electrically neutral form, when then it is converted by intracellular esterases, which are active only in entire cells, into the negatively charged, green fluorescent calcein [18]. When plasma membrane becomes compromised, esterase activity ceases and ethidium homodimer (EthD-1) can react with nucleic acids, producing red fluorescence [19]. Thus, calcein-AM allows determination of cellular viability through esterase activity decrease, while EthD-1 allows verifying the physical and chemical alterations in the cellular membrane, with elevation of 40 times in its fluorescence when associated to nucleic acids. In previous studies, calcein-AM proved to be specific and sensitive for detection and tracking of apoptosis in living cells, as PC 12 and NIH3T3 [19,20]. Considering the fact that these studies were performed with adherent cells and did not 
include mononuclear cells, such as lymphocytes, the present study compared the performances of calcein-AM and ethidium homodimer (EthD-1) on apoptosis and cellular viability quantification and detection from peripheral blood mononuclear HIV-infected cells, maintained in RPMI1640 during 24 and 48 hours, with annexin V-FITC and propidium iodide, which is widely employed in cytometry as an early marker for apoptosis.

\section{Material and Methods}

Blood Samples

Cellular viability and apoptosis of mononuclear cells were simultaneously evaluated in blood samples from $50 \mathrm{HIV}$ infected patients who are routinely assisted at the Hospital of the Federal University of Santa Catarina and submitted to antiretroviral therapy. After signing the informed consent, patients who accepted to participate in this study were submitted to additional peripheral blood sample collection (50 mL) by antecubital puncture with a heparinized tube (sodic heparin), using vacuous system. Selection of patients was conditioned to the absence of opportunistic infections. This study was previously approved by Ethics Committee for Human Research of the Federal University of Santa Catarina (UFSC-protocol 163/2003).

\section{Mononuclear Cells Isolation}

The mononuclear cells were separated by density gradient utilizing Ficoll-Hypaque (density 1077) (Sigma Chemical Company, Saint Louis, USA). After blood centrifugation at 1,500 rpm (378 g) for 15 minutes; the formed layer of white globules between the plasma and the red cells was removed and resuspended in $8 \mathrm{~mL}$ of phosphate buffered saline $0.01 \mathrm{M}$ (PBS), pH 7.4. The cell suspension containing leucocytes and red globules was gently transferred to another tube with 2.5 $\mathrm{mL}$ of Ficoll-Paque and centrifuged at 1,500 rpm (378 g). The formed cell layer between PBS and Ficoll-Paque was collected and washed twice in PBS, $\mathrm{pH}$ 7.4. Mononuclear cells were resuspended and the cell suspension was adjusted to a concentration of 5 X $10^{5}$ cells/mL in RPMI 1640 medium with $25 \mathrm{mM}$ HEPES and $2 \mathrm{mM}$ L-glutamine (Sigma Chemical Company, Saint Louis, USA), supplemented with $10 \%$ heatinactivated fetal calf serum (Sigma Chemical Company, Saint Louis, U.S.A.), $10 \mathrm{UI} / \mathrm{mL}$ penicillin, $10 \mu \mathrm{g} / \mathrm{mL}$ streptomycin and $5 \mu \mathrm{L}$ amphotericin $\mathrm{B}$.

\section{Mononuclear Cells Maintenance}

Mononuclear cells (5 X 10 ${ }^{5}$ ) were kept in $2.0 \mathrm{~mL}$ of RPMI 1640 medium with 25 mM HEPES and 2 mM L-glutamine (Sigma Chemical Company, Saint Louis, USA), supplemented with $10 \%$ heat-inactivated fetal calf serum (Sigma Chemical Company, Saint Louis, USA), $10 \mathrm{UI} / \mathrm{mL}$ penicillin, $10 \mu \mathrm{g} / \mathrm{mL}$ streptomycin and $5 \mu \mathrm{L}$ amphotericin B. From the 50 blood samples obtained, 25 were kept as described during 24 hours, while the other 25 samples were kept during 48 hours, both at $37^{\circ} \mathrm{C}$ in a $5 \% \mathrm{CO}_{2}$ atmosphere.
Lymphocytes Viability and Apoptosis Detection Assays

The determination of viable and apoptotic lymphocytes was performed using the Annexin V-FITC apoptosis detection kit [containing annexin V-FITC, propidium iodide and buffer (SIGMA Chemical Co. Saint Louis, MILLSTONE, USA)] and Live/Dead viability and cytotoxicity detection kit (L-3224), containing calcein-AM and ethidium homodimer-1 (Molecular Probes Incorporation, Eugene, Oregon, USA). The lymphocytes viability and apoptosis were determined by FACSCallibur (Becton Dickinson Immunocytometry Systems, San Jose, CA, USA) flow cytometer, through the CELLQuest and PAINT-A-GATE programs.

\section{Statistical Analysis}

Statistical analysis was based on paired t tests for comparison between the mean rates obtained, which studied the effect of the marker type on viable and apoptotic lymphocytes classification after 24 and 48 hours of incubation. $\mathrm{p}<0.05$ was considered significant using the softwares SAS ${ }$ System and Statistica ${ }^{\circledR} 6.0$.

\section{Results}

Mononuclear Cells Acquisition

After mononuclear cells acquisition by FACSCallibur flow cytometer (Becton Dickinson. USA), through CELLQuest (Figure 1) and PAINT-A-GATE (Figure 2) programs, cells were selected according to their volume and granularity. Thus, the larger mononuclear cells as monocytes, and granulocytes, which were not separated by Ficoll-Paque, could be excluded.

The CELLQuest program allows distinguishing three different lymphocyte groups (Figures 3 and 4). On the other hand, the PAINT-A-GATE program allows distinguishing three different lymphocyte groups, which were selected and colored. Therefore, the analyses through Annexin V-FITC apoptosis detection kit by PAINT-A-GATE program showed that lymphocytes strongly stained with annexin, located on the right medial region, corresponded to apoptotic cells. Lymphocytes strongly stained with propidium iodide, located on the right higher region, corresponded to death cells. Finally, lymphocytes not stained with annexin or propidium iodide, located on the lower left region, corresponded to viable cells (Figure 5).

Analyses through Live/Dead viability and cytotoxicity detection kit by PAINT-A-GATE program showed that lymphocytes strongly stained with calcein, located on the lower right region, corresponded to viable cells. Lymphocytes strongly stained with ethidium homodimer, located on the higher region, corresponded to death cells. Finally, lymphocytes weakly stained with calcein and propidium iodide, located on the medial region, corresponded to apoptotic cells (Figure 6). Therefore, the rates of each lymphocyte group were determined and the mean percentage values were calculated. 
Figure 1. Selected cells and/or structures through CELLQuest program, according to their volume and granularity.

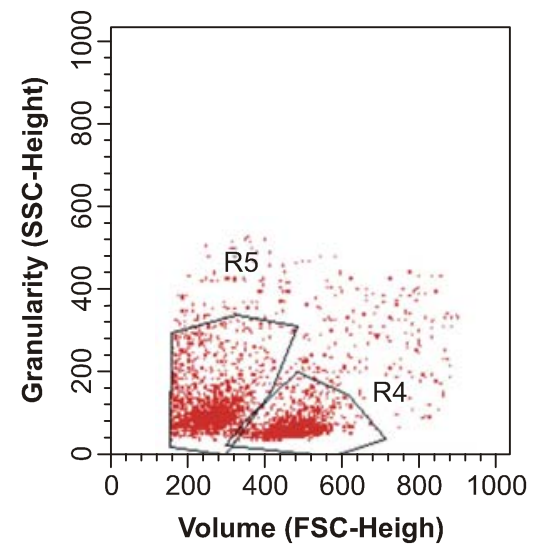

Figure 2. Selected and analyzed cells through PAINT-AGATE, according to their volume and granularity.

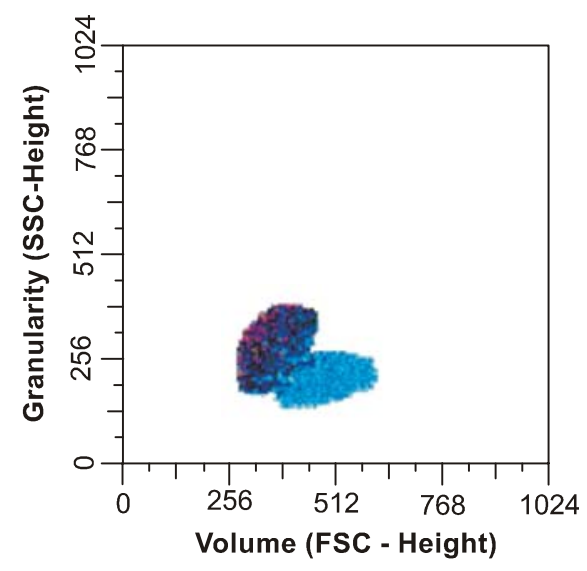

Figure 3. Lymphocytes distribution, obtained through CELLQuest, according to stain intensity by annexin V-FITC and/or by propidium iodide.

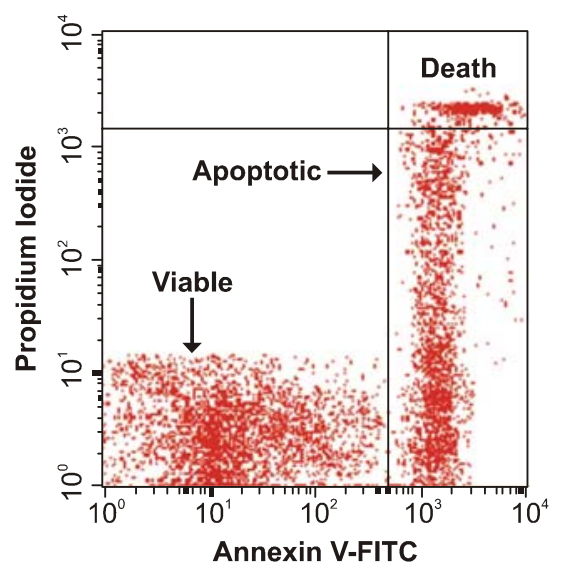

Figure 4. Lymphocytes distribution, obtained through CELLQuest, according to stain intensity by calcein-AM and/ or by ethidium homodimer.



Figure 5. Lymphocytes distribution, obtained through PAINTA-GATE, according to stain intensity by annexin V-FITC and/ or by propidium iodide.

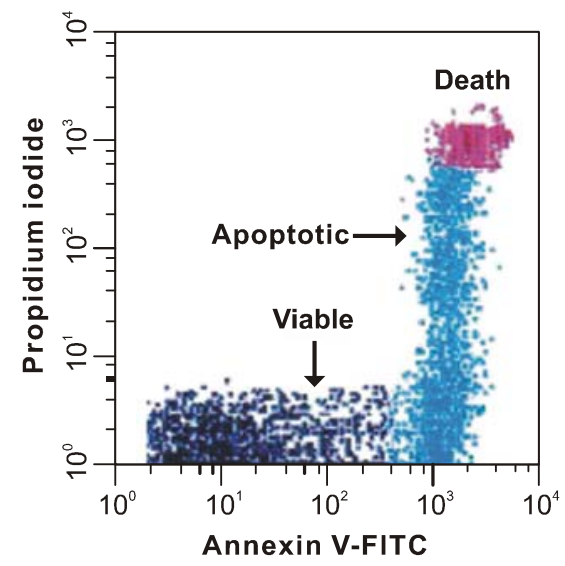

Figure 6. Lymphocytes distribution, obtained through PAINTA-GATE, according to stain intensity by calcein-AM and/or by ethidium homodimer.

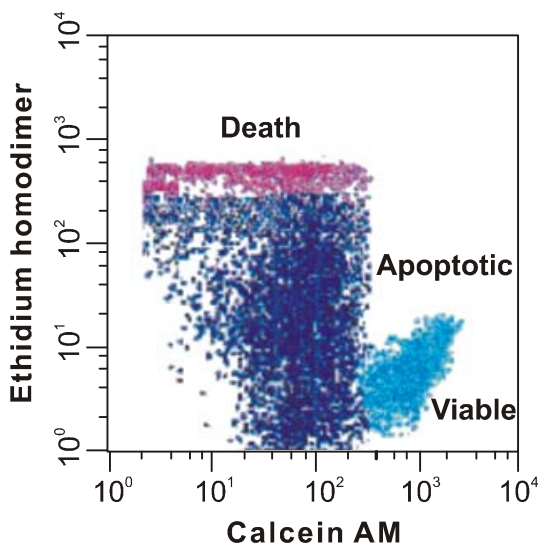


Correlations Between the Results Obtained with Annexin VFITC/Ethidium Homodimer and Calcein-AM/Propidium iodide After 24 and 48 Hours of Incubation

The determination of the Pearson's correlation coefficients demonstrated that viability and apoptotic cellular levels, obtained by the calcein-AM/ethidium homodimer staining after 24 hours, presented significant correlations among the levels of viable $(\mathrm{r}=0.9517, \mathrm{p}<0.001)$ and apoptotic lymphocytes $(\mathrm{r}=0.9681, \mathrm{p}<0.001)$ obtained by the annexin $\mathrm{V}$ FITC/propidium iodide staining after 24 hours of incubation.

After 48 hours of incubation, the Pearson's correlation coefficients demonstrated that viability and apoptotic cellular levels, obtained by the calcein-AM/ethidium homodimer staining after 48 hours, presented significant correlations among the levels of viable $(r=0.9557 ; p<0.001)$ and apoptotic lymphocytes $(r=0.9160, p<0.001)$ obtained by the annexin $V$ FITC/propidium iodide staining after 48 hours of incubation.

\section{Quantification of Viable and Apoptotic Lymphocytes After} 24 Hours of Incubation

The determination of cellular viability levels after 24 hours of incubation did not demonstrate significant difference $(\mathrm{p}=$

Figure 7. Mean viable cell rates obtained by annexin V-FITC/ propidium iodide and calcein-AM and ethidium homodimer $(n=25)$ after 24 hours of incubation.

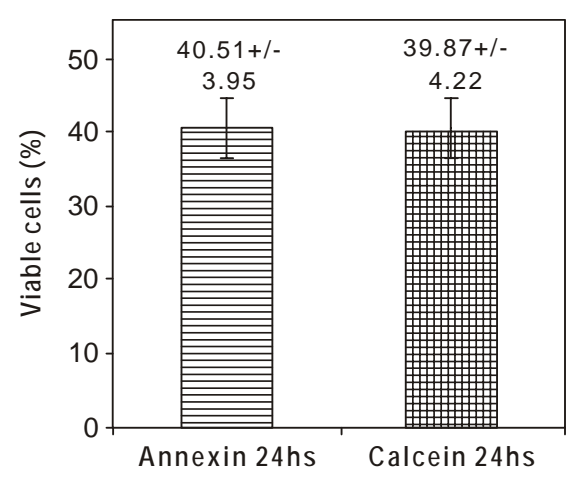

Figure 8. Mean apoptotic cell rates obtained by annexin VFITC/propidium iodide and calcein AM/ethidium homodimer $(n=25)$ after 24 hours of incubation.

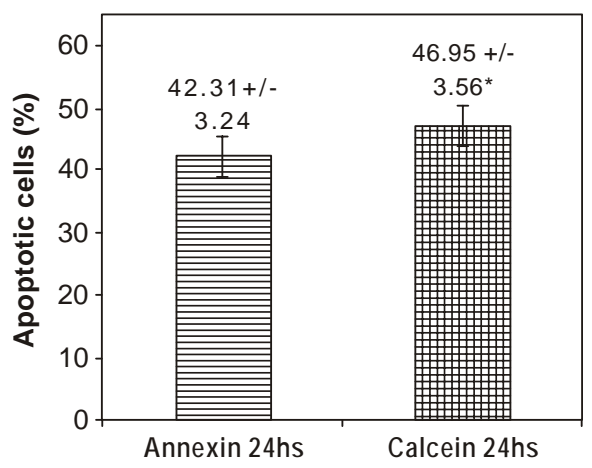

0.6284 ) among the mean viable lymphocyte rates, obtained by annexin V-FITC/PI (40.51\% \pm 3.95$)$ and calcein-AM/EthD-1 $(39.87 \% \pm 4.22)$ (Figure 7). The evaluation of apoptosis levels after 24 hours of incubation demonstrated that the mean apoptotic lymphocyte rates obtained by calcein-AM/EthD-1 $(46.95 \% \pm 3.65)$ were statistically superior $(p<0.0001)$ when compared to the values obtained by annexin V-FITC/PI (42.31\% \pm 3.24 ) (Figure 8).

Quantification of Viable and Apoptotic Lymphocytes After 48 Hours of Incubation

The determination of cellular viability levels after 48 hours of incubation did not demonstrate significant difference $(\mathrm{p}=$ 0.4290 ) among the mean viable lymphocyte rates, obtained by annexin V-FITC/PI $(40.24 \% \pm 4.06)$ and calcein-AM/EthD-1 $(41.22 \% \pm 4.10)$ (Figure 9).

The determination of apoptotic levels after 48 hours of incubation time demonstrated that the mean percentage values of apoptotic lymphocyte rates obtained by calcein-AM/EthD$1(37.67 \% \pm 2.47)$ were statistically superior $(p=0.0014)$ when compared to the values obtained by annexin V-FITC/PI (33.83\% \pm 2.65 ) (Figure 10).

Figure 9. Mean viable cell rates obtained by annexin V-FITC/ propidium iodide and calcein-AM/ethidium homodimer ( $\mathrm{n}=$ 25) after 48 hours of incubation time.

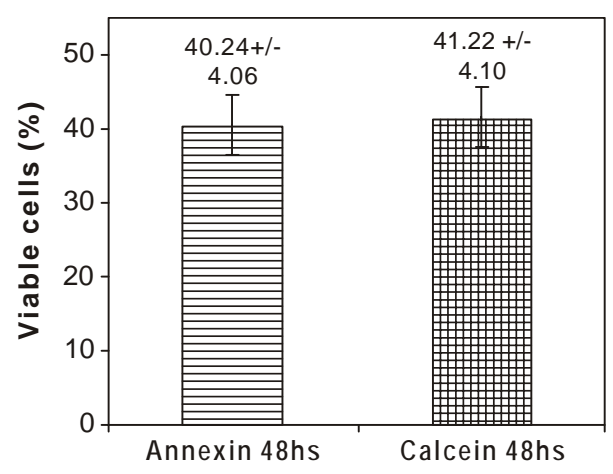

Figure 10. Mean apoptotic cell rates obtained by annexin VFITC/propidium iodide and calcein AM/ethidium homodimer $(n=25)$ after 48 hours of incubation.

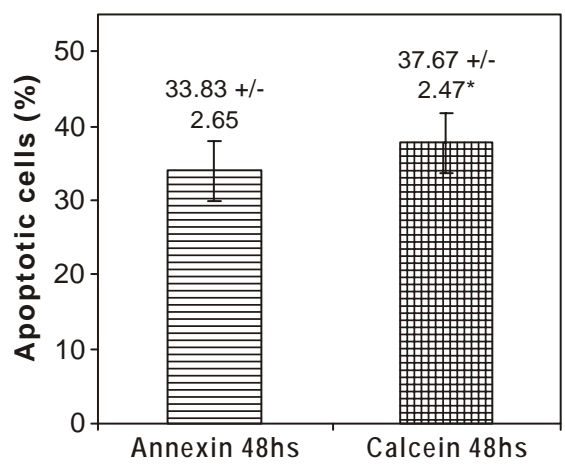




\section{Discussion}

Apoptosis can be differentiated from necrosis through the observation of certain morphologic alterations, which are different and peculiar to each cellular death process [17]. Whereas shrinkage of the cytoplasm, chromatin condensation, membrane blebbing and formation of apoptotic bodies characterize apoptosis; the cellular swelling and karyolysis typically occur in necrosis [21]. Besides, metabolic derangements of necrosis lead to osmotic lesions of cell membrane which are, instead, substantially unaffected by apoptotic process [20].

The abnormal and disordered regulation of apoptosis can be involved in the pathogenesis of several disorders, like cerebral and myocardial ischemia, autoimmune and neurodegenerative diseases, organ and bone marrow transplant rejections, or viral infections, including HIV infection [22].

Although the exact role of apoptosis during HIV infection remains to be defined, growing evidences suggest that apoptosis is a major contributor for the depletion of the CD4+ lymphocytes in vivo [23-26]. The recent recognition that programmed cell death is an important feature of CD4+ T cells depletion during HIV infection stimulates the viability verification of assays thoroughly employed on mononuclear apoptosis quantification [27].

The phosphatidylserine exposure on the surface of apoptotic cells is a general apoptosis feature occurring before membrane bleb formation and internucleosomal DNA cleavage $[28,29]$. Annexin V-FITC is presently employed in evaluation and quantification of cellular apoptosis by flow cytometry due to its specific bind to phosphatidylserine residues $[14,15,30]$. Thus, viable cells are classified as annexin V negative, while the apoptotic cells are annexin $\mathrm{V}$ positive [31]. In addition, the concomitant uses of nuclear fluorescent dye propidium iodide makes possible to verify the characteristic nuclear alterations of the late apoptosis stages, since the membrane permeability increases.

The progressive increase of plasma membrane permeability, on the other hand, comes as a typical morphologic alteration of late apoptotic or death cells [32]. This permeability increase allows the entrance and permanence of toxins in the cytoplasm, which in turn, promote the intracellular esterasic activity decrease. Therefore, the intracellular esterasic activity levels could be used both as a probe of viability and plasma membrane competence and as an indicator of the cellular functionality [20].

The analysis of the cellular death processes by flow cytometry performed in the present study turned possible the accurate quantitative detection of viable cellular populations and apoptotic cells (Figures 1 and 2). Thus, through CELLQuest and PAINT-A-GATE programs, lymphocytes were differentiated and acquired according to their size (forward scatter-FSC) and granularity (side scatter - SSC) (Figures 36). As expected, the mean apoptosis rates both after 24 and 48 hours were significantly superior when the cells were treated with calcein-AM and ethidium homodimer (Figures 8 and 10). On the other hand, the mean viable cell rates were not significantly different between calcein-AM/ethidium homodimer and annexin V-FITC/propidium iodide treatments for both incubation periods.

As could be observed by apoptosis levels demonstrated by the annexin V-FITC (mean of $42.31 \% \pm 3.24$ after 24 hours; Mean of $33.83 \% \pm 2.65$ after 48 hours), the mononuclear cells, as well as other cell lineages previously determined by this marker $[4,33]$, equally exhibit phosphatidylserine residues in the outer leaflet of the plasma membrane lipid bilayer. However, the results demonstrated that the method that uses annexin V-FITC and propidium iodide was less sensitive for evaluation and quantification of apoptotic mononuclear cells when compared to calcein-AM and ethidium homodimer markers in HIV infection.

Previous studies showed that cells of a same cellular lineage can undergo apoptosis without demonstrating the concomitant phosphatidylserine translocation [34,35]. It appears unlikely that phospholipids asymmetry, if lost at the very beginning of the process, could be restored later, because of the depletion of energetic resources occurring in the late stages of apoptosis. Thus, it is more conceivable that between the studied apoptotic cells, some of them have not presented phosphatidylserine translocation, and consequently, could not be stained by the annexin V-FITC.

The reduced sensibility of this marker when compared to calcein-AM could be yet related to the fact that the phosphatidylserine translocation, which forms the basis for apoptosis detection by annexin V-FITC, can occur in necrotic cells also [30,36]. Moreover, even if phosphatidylserine translocation do not occur during necrosis, loss of plasma membrane integrity, which characterizes necrosis, can allow easy access of the detection reagent to the membrane interior resulting in false positive staining [27].

Besides, Lecoeur \& Gougeon [32] demonstrated that the mean apoptosis rates observed on HIV-infected lymphocytes quantified by iodide were underestimated due to the granulocytes interference in the propidium iodide-dependent apoptosis assay. This interference is due to the enrichment of granulocytes associated to HIV infection, observed in the mononuclear cells composition following Ficoll-Paque isolation.

In fact, the positive or negative interference of granulocytes in propidium iodide-dependent apoptosis assay depends on the propensity of these cells to undergo spontaneous apoptosis following short term-culture [37]. During HIV infection, both eosinophils and neutrophils might be involved in apoptosis process, promoting the lymphocytes apoptosis rate reduction [32]. Thus, the uses of annexin VFITC and propidium iodide present limitations that could supply biases in apoptosis determination and quantification which, in turn, must be considered during the choice of sensitive methodologies to evaluate this cellular death process. 
A previous study in different adherent cells, as PC 12 and NIH3T3, demonstrated that acetoxymethyl ester of calcein (calcein-AM) and ethidium homodimer (EthD-1) were more sensitive in earlier detection of apoptosis when compared to annexin V-FITC/propidium iodide [35]. In fact, the present study demonstrated that calcein-AM/ethidium homodimer was equally useful and more sensitive in mononuclear cell apoptosis quantification, when compared to annexin V-FITC/propidium iodide; besides allowing clarity when defining the viable and apoptotic lymphocyte populations.

Such verifications results from calcein-AM inherent characteristics, which provides morphological evidences of chromatin condensation and segregation in blebs [38]. However, this marker is quickly liberated from the cellular cytoplasm as the plasma membrane becomes committed, even in the presence of residual intracellular esterase activity [20].

On the other hand, functional information regarding plasma membrane competence and dead cells can be obtained through ethidium homodimer, which accumulates in DNA fragments of damaged plasma membrane cells, but could not penetrate in the entire plasma membrane cells [39].

In the present study, the viability and apoptosis levels of mononuclear cells obtained by calcein-AM/ethidium homodimer demonstrated significant correlations among the levels of the same cellular groups obtained by annexin VFITC/propidium iodide, after 24 and 48 hours of incubation. This verification validates the hypothesis that both markers allow evaluating the viability and apoptosis of $\mathrm{T}$ lymphocytes in a similar way, although their sensibilities in apoptotic cells quantification were different.

The growing recognition of apoptosis as an important pathogenic characteristic of several disorders, including HIV infection progression, has been increasing further than the burgeoning interest in apoptosis research. Although it is clear that apoptosis exacerbation or its inhibition is involved in disease symptoms, there is much room for intensive research to arrive at a deeper understanding. Therefore, techniques that allow for accurate and quantitative detection of apoptosis need constant evaluations and improvement.

\section{Acknowledgements}

The authors thank Maria de Lourdes Rovaris, M.D., and the staff of the Laboratory of Clinical Analysis of the Hospital the Federal University of Santa Catarina, for patient support; Maria Cláudia Santos, Ph.D., Maria Luiza Bazzo, M.D., Tânia Beatriz Creczynski Pasa, Ph.D., for laboratory experiment support, Paulo José Ogliari, Ph.D. and Juliano Anderson Pacheco, M.D., for statistical analysis. This study was supported by FUNPESQUISA.

\section{References}

1. Kerr J.F.R., Wyllie A.H., Currie A.R. Apoptosis: a basic biological phenomenon with wide-ranging implications in tissue kinetics. Br J Cancer 1972;26:239-57.
2. Batistou A., Greene L.A. Internucleosomal DNA cleavage and neoronal cell survival/death. J Cell Biol 1993;122:523-532.

3. Van Engeland M., Kujipers H.J.H., Ramaekers F.C.S., et al. Plasma membrane alterations and cytoskeletal changes in apoptosis. Exp Cell Res 1997;235:421-30.

4. Kimura E., Aoki S., Kikuta E., et al. A macrocyclic zinc(II) fluorophore as a detector of apoptosis. Proc Natl Acad Sci USA 2003;100:3731-6.

5. Saikumar P., Dong Z., Mikailow V., et al. Apoptosis: definition, mechanisms, and relevance to disease. Am J Med 1999; $107: 489-506$.

6. Nagata S. Fas-mediated apoptosis. Adv Exp Med Biol 1996; $406: 119-24$.

7. Salmena L., Lemmers B., Hakem A., et al. Essential role for caspase 8 in T-cell homeostasis and T-cell-mediated immunity. Genes Dev 2003;17:883-95.

8. Thompson C.B. Apoptosis in the pathogenesis and treatment of diseases. Science 1995;26:1456-62.

9. Duke R.C., Ojcius D.M., Young D.E. Cell suicide in health and disease. Sci Am 1996;275:80-7.

10. Nicoletti I., Migliorati G., Pagliacci M.C., et al. A rapid and simple method for measuring thymocyte apoptosis by propidium iodide staining and flow cytometry. J Immunol Methods 1991;139:271-9.

11. Petit P.X., Lecoeur H., Zorn E., et al. Alterations in mitochondrial structure and function are early events of dezamethasone-induced thymocyte apoptosis. J Cell Biol 1995;130:157-67.

12. Brown D.G., Sun X.M., Cohen G.M. Dexamethasone-induced apoptosis involves cleavage of DNA to large fragments prior to internucleosomal fragmentation. $\mathrm{J}$ Biochem Chem 1993;268:3037-9.

13. Koopman G., Reutelingsperger C.P.M., Kuijten G.A.M., et al. Annexin $\mathrm{V}$ for flow cytometic detection of phosphatidylserine expression on B cells undergoing apoptosis. Blood 1994;84:1415-20.

14. Clarke R.G., Lund E.K., Johnson I.T., Pinder A.C. Apoptosis can be detected in attached colonic adenocarcinoma HT29 cells using annexin $\mathrm{V}$ binding, but not by TUNEL assay or sub-G0 DNA content. Cytometry 2000;39:141-50.

15. Lee W.D., Flynn A.N., LeBlanc, J.M., et al. Tilmicosin-induced bovine neutrophil apoptosis is cell-specific and downregulates spontaneous LIB4 synthesis without increasing Fas expression. Vet Res 2004;35:213-24.

16. Van Engeland M., Nieland, L.J., Ramaekers F.C.S., et al. Annexin V-affinity assay: a review on an apoptosis detection system based on phosphatidylserine exposure. Cytometry 1998;31:1-9.

17. Willingham M.C. Cytochemical methods for the detection of apoptosis. J Histochem Cytochem 1999;47:1101-9.

18. Neri S., Mariani E., Meneghetti A., et al. Calcein-acetoxymethyl cytotoxicity assay: Standardization of a method allowing additional analyses on recovered effector cells and supernatants. Clin Diagn Lab Immunol 2001;8:1131-5.

19. Gatti R., Belletti S., Orlandini G., et al. Comparison of annexin V and calceina-AM as early vital markers of apoptosis in adherent cells by confocal laser microscopy. J Histochem Cytochem 1998;46:895-900

20. Bevers E.M., Comfurius P., Zwall R.F.A. Regulatory mechanisms in maintenance and modulation of transmembrane lipid asymmetry: pathophysiological implications. Lupus 1996;5:480-7.

21. Bussolati O., Belletti S., Uggeri J., et al. Characterization of apoptotic phenomena induced by treatment with L-Asparginase in NIH3T3 cells. Exp Cell Res 1995;220:283-91.

22. Kannan K., Jain S.K. (2000) Oxidative stress and apoptosis. Pathophysiology 1995; 7:153-63.

23. Ahr B., Robert-Hebmann V., Devaux C., et al. Apoptosis of uninfected cells induced by HIV envelope glycoproteins. Retrovirology 2004;1:1-12. 
24. Badley A.D., Dockrell D., Paya C.V. Apoptosis in AIDS. Adv Pharmacol 1997;41:271-94.

25. Patel C.A., Mukhtar M., Pomerantz R.J. Human Immunodeficiency Virus type $1 \mathrm{Vpr}$ induces apoptosis in human neuronal cells. J Virol 2000;74:9717-26.

26. Lum J.J., Cohen O.J., Nie Z., et al. VprR77Q is associated with long-term nonprogressive HIV infection and impaired induction of apoptosis. J Clin Invest 2003;111:1547-54.

27. Chaves M.M., Kallas E.G. Cell cycle distribution of CD4+ lymphocytes in HIV-1-infected subjects. Cytometry 2004;62B:46-51.

28. Martin S.J., Reutelingsperger C.P., McGahon A.J., et al. Early redistribution of plasma membrane phosphatidylserine is a general feature of apoptosis regardless of the initiating stimulus: inhibition by over expression of Bcl-2 and Abl. J Exp Med 1995; $182: 1545-56$.

29. Blankenberg F.G., Katsikis P.D., Tait J.F., et al. In vivo detection and imaging of phosphatidylserine expression during programmed cell death. Proc Natl Acad Sci USA 1998;95:6349-54.

30. Lassus P., Hibner U. Detection and quantification of apoptosis in transiently transfected adherent cells. Nucleic Acids Research 1998;26:5233-4.
31. Lecoeur H., Gougeon M.L. Comparative analysis of flow cytometric methods for apoptosis quantization in murine thymocytes and human peripheral lymphocytes from controls and HIV-infected persons. Evidence for interference by granulocytes and erythrocytes. J Immunol Methods 1996;198:87-99.

32. Frey T. Correlated flow cytometric analysis of terminal events in apoptosis reveals the absence of some changes in some model systems. Cytometry 1997;28:253-63.

33. Vermes I., Haanen C., Steffens-Nakken H., et al. A novel assay for apoptosis. Flow cytometric detection of phosphatidylserine expression on early apoptotic cells using fluorescein labelled annexin V. J Immunol Methods 1995;184:39-51.

34. Stern M., Meagher L., Savil J., et al. Apoptosisin human eosinophils. Programmed cell death in the eosinophil leads to phagocytosis by macrophages and is modulated by IL-5. J Immunol 1992;148:3543-9.

35. Kato M., Makino S., Kimura H., etal. Evaluation of mitochondrial function and membrane integrity by dual flourescent staining for assessment of sperm status in rats. J Toxicol Sci 2002;27:11-8.

36. Chen C.C., Chang J.H., Lee J.B., Human corneal epithelial cell viability and morphology after dilute alcohol exposure. Invest Opht Vis Sci 2002;43:2593-602. 